\title{
Impact of Interconnect Multiple-Patterning Variability on SRAMs
}

\author{
Ioannis Karageorgos*† Michele Stucchi* Praveen Raghavan* Julien Ryckaert* Zsolt Tokei* \\ Diederik Verkest* Rogier Baert* Sushil Sakhare* Wim Dehaene ${ }^{\dagger *}$ \\ * imec, Kapeldreef 75, B-3001 Leuven, Belgium \\ $\dagger$ KU Leuven, ESAT, B-3001 Leuven, Belgium \\ email: ioannis.karageorgos@imec.be
}

\begin{abstract}
The introduction of Multiple Patterning (MP) in sub-32nm technology nodes may pose severe variability problems in wire resistance and capacitance of IC circuits. In this paper we evaluate the impact of this variability on the performance of SRAM cell arrays based on the $10 \mathrm{~nm}$ technology node, for a relevant range of process variation assumptions. The MP options we consider are the triple Litho-Etch $\left(\mathrm{LE}^{3}\right)$ and the Self Aligned Double Patterning (SADP), together with Single Patterning Extreme-UV (EUV). In addition to the analysis of the worst-case variability scenario and the impact on SRAM performance, we propose an analytical formula for the estimation of SRAM read time penalty, using the $\mathrm{RC}$ variation of the bit line and the array size as input parameters. This formula, verified with SPICE simulations, allows a fast extraction of the statistical distribution of the read time penalty, using the Monte-Carlo method. Results on each patterning option are presented and compared.
\end{abstract}

\section{INTRODUCTION}

As the semiconductor industry struggles to keep pace with Moore's Law, it faces ever increasing challenges. Many of these challenges are lithography related [1]. Dry and later immersion lithography at $193 \mathrm{~nm}$ drove successfully the semiconductor roadmap down to $45 \mathrm{~nm}$ node [2]. However, as the resolution of photoresist patterns begins to blur at around 45 $\mathrm{nm}$ half-pitch [3], a single lithography exposure is not sufficient for printing the denser features of the next technology nodes. The two most apparent solutions to overcome this issue can be either the transition to a shorter wavelength source lithography, as in Extreme-UV (EUV), or the use of Multiple Patterning (MP) which is an enhanced lithography process that produces multiples of the minimum-sized features. However, EUV has been confronted with a series of materials and source issues, making it not yet a manufacturable solution. As a result, the use of MP in optical lithography currently appears the only viable solution for further technology scaling [4], [5]. In the semiconductor industry, double patterning was introduced for the $32 \mathrm{~nm}$ half-pitch node and beyond [2], allowing double feature density with the incumbent technology.

There are several technology options available for double or multiple patterning [6]. Yet, the two most viable and widely adopted options in industry for the $10 \mathrm{~nm}$ technology node (imec N10 node), which are considered also in this paper, are: the Self Aligned Double Patterning (SADP) and the triple Litho-Etch approach (LELELE) [5], [7], [8].

The use of MP options in Back-End-of-Line (BEOL) introduces new variability challenges in wires. MP variations can lead to different geometries and spacing in arrays of parallel wires, which results in variations in resistance, capacitance and coupling-capacitance. This leads to a performance unbalance in supposedly identical parallel wires and may pose problems in circuits sending and receiving signals through these parallel interconnects. The continuous reduction of interconnect dimensions predicted by the scaling roadmap [9] can only exacerbate these problems.

In this study we evaluate the impact of MP options on the performance of SRAMs based on the N10 technology node. The assessment includes the comparison of multiple Litho-Etch and SADP options together with Single Patterning (SP) EUV for a relevant range of process variation. The content of this paper is organized as follows: Section II covers the impact of worst case variability on SRAM read time, using time-to-discharge of bit lines $(t d)$ as the figure of merit (FOM). In Section III a simple analytical formula is proposed for the estimation of $t d$ penalty as a result of bit line $R C$ variations. Based on the formula, we present a comparison of $t d$ penalty distribution for each patterning option using MonteCarlo sampling of process variation as input. The conclusions of this study are presented in Section IV.

\section{SRAM WORST CASE VARIABILITY STUDY}

The variability assessment presented in this section is based on the six-transistor (6T) SRAM cell (Fig. 1a). As a target layout we used a high-density optimized SRAM cell design, developed at imec for the N10 node (Fig. 1b). This layout uses unidirectional $(\mathrm{u} / \mathrm{d})$ horizontal metall at minimum spacing for the bit lines and power grid and $\mathrm{u} / \mathrm{d}$ vertical metal 2 for the word lines. The three options we consider for metall patterning are: $\mathrm{LE}^{3}$, SADP (because of $\mathrm{u} / \mathrm{d}$ arrangement, layout can be easily derived with double patterning SADP) and single patterning EUV. The top view of metall layout and the patterning details for each option are shown in Fig. 2.

\section{A. Simulation environment}

This analysis is based on SPICE-level simulations of the SRAM cell array including the N10 transistor compact models. The netlists with parasitics were extracted from the SRAM array layout by a parameterized Layout Parasitic Extraction (LPE) tool, developed at imec and based on the standard LPE tool flow but adapted to include process variability. The tool 


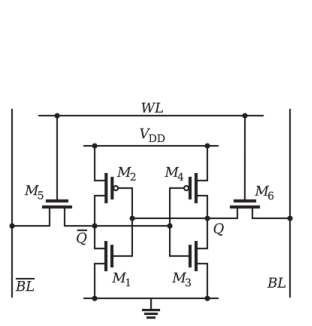

(a) Schematic

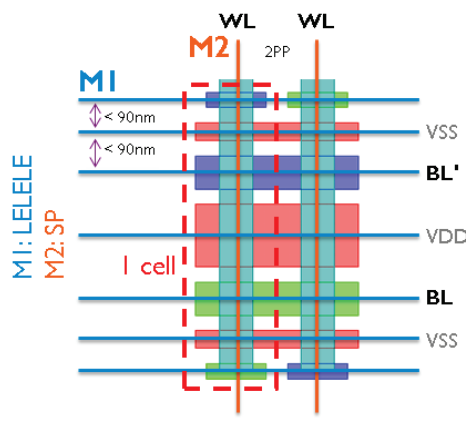

(b) Layout
Fig. 1: 6T SRAM cell circuit and layout.

inputs are technology parameters (layer thickness, tapering angles, material properties, etch and CMP parameters) and MP-related layer operations (CD, overlay and spacer thickness variation) together with the target layout (GDSII); the tool generates the LPE deck, runs the simulations and stores the target metrics $(R, C, C C)$ or netlists (with parasitics), in an iterative loop. The implementation of Monte-Carlo sampling of the input variability parameters allows the extraction of the $R C$ distribution for any wire (e.g. the bit line).

The technology parameters and the MP process variation assumptions we use in our simulations are based on in-house experimental data. The most relevant of these assumptions are the following:

- $3 \sigma \mathrm{CD}$ variation of $3 \mathrm{~nm}$ for $\mathrm{LE}^{3}$, SADP core layer and EUV (although this value may be pessimistic for EUV)

- $3 \sigma$ SADP spacer variation of $1.5 \mathrm{~nm}$

- $3 n m$ to $8 n m$ range of $3 \sigma$ OL error for $\mathrm{LE}^{3}$

- metal1_B and metal1_C are aligned to metal1_A for $\mathrm{LE}^{3}$

- spacer-defined bit lines for SADP (Fig. 2:SADP)

\section{B. Variability worst cases for $C_{b l}$}

Our simulation results show that bit line capacitance $\left(C_{b l}\right)$ is the dominant parameter affecting the SRAM performance, even for long arrays (up to 512 cells). The resistance of bit lines is relatively low due to the non-minimum $\mathrm{CD}$ of bit line wires, which is typical in SRAM. We focus on metall layer since this is the layer of the bit lines in this design and because it is often the most critical interconnect layer [10]. Initially we consider the extreme case of $8 \mathrm{~nm} 3 \sigma \mathrm{OL}$ error for $\mathrm{LE}^{3}$. Using all combinations of $\mathrm{CD}$ and OL errors as input parameters, we identified the worst case scenario for each option with respect to $C_{b l}$ increase. The results are listed in Table I. Patterning details and the consequent layout distortion for each case are shown in Fig. 2. Each color of $\mathrm{LE}^{3}$ in Fig. 2 represents a different patterning step and 'plus', 'minus' signs refer to $3 \sigma$ values.

The impact of $\mathrm{LE}^{3}$ worst case variability on $C_{b l}$ is quite high. On the other hand, for SADP, because of the self-aligned nature of the process, the impact is limited and even smaller than EUV (assuming the same $3 n m 3 \sigma \mathrm{CD}$ in EUV).

\section{Array size \& worst case td variation}

The impact of $R C_{b l}$ variation on $t d$ is also related to the size of the array (since the FEOL resistance path doesn't scale with
TABLE I: Worst case variability for each patterning option.

\begin{tabular}{lllll}
\hline Pat. option & Param & Layer & Value & $\mathrm{R} \&$ C impact \\
\hline \multirow{4}{*}{ LELELE } & $\mathrm{CD}$ & metal1_A & $+3 \sigma$ & \\
& $\mathrm{CD}$ & metal1_B & $+3 \sigma$ & $C_{b l}:+61.56 \%$, \\
& $\mathrm{CD}$ & metal1_C & $+3 \sigma$ & $R_{b l}:-10.36 \%$ \\
& OL(vert.) & metal1_B & $-3 \sigma$ & \\
& OL(vert.) & metal1_C & $+3 \sigma$ & \\
\hline \multirow{2}{*}{ SADP } & CD & metal1_core & $-3 \sigma$ & $C_{b l}:+4.01 \%$, \\
& CD & metal1_spacer & $-3 \sigma$ & $R_{b l}:-18.19 \%$ \\
\hline \multirow{2}{*}{ EUV } & CD & metal1_A & $+3 \sigma$ & $C_{b l}:+6.65 \%$, \\
& & & & $R_{b l}:-10.36 \%$ \\
\hline
\end{tabular}

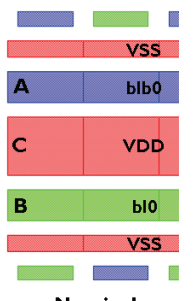

Nominal

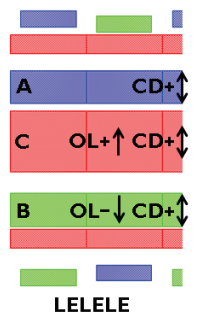

LELELE

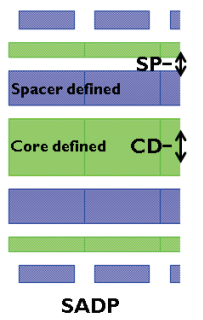

SADP

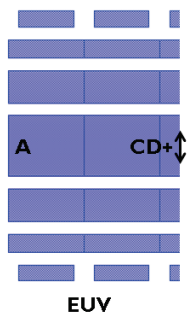

EUV
Fig. 2: Worst case variability \& metal1 layout distortion.

array size). In our Design of Experiments (DOE) we consider four different SRAM array sizes of 16 word lines (wl), $64 \mathrm{wl}$, $256 \mathrm{wl}$ and $1024 \mathrm{wl}$ and a fixed number of 10 bit line (bl) pairs (or 10bit word-length). An overview of this arrangement is shown in Fig. 3. The length of bl is proportional to the number of wl. We keep the bl count fixed because it is not relevant for this study; their number is large enough to consider the simulation results of the central lines not affected by edge related effects.

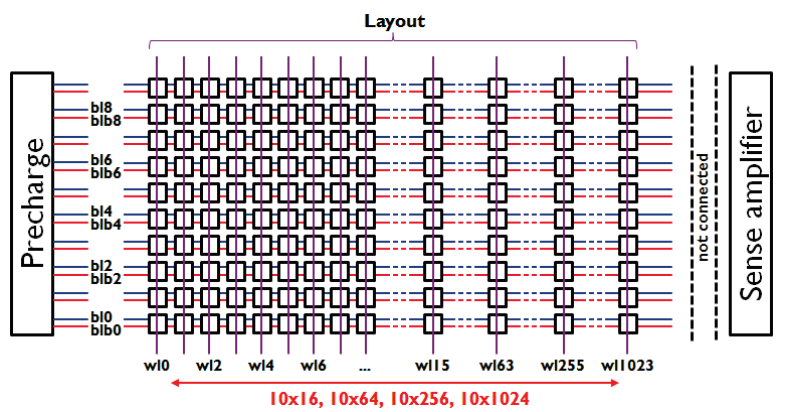

Fig. 3: Overview of the SRAM arrays used in simulations.

Our simulation assumptions include:

- vdd, precharge, wl enable: $0.7 \mathrm{~V}$

- sense amplifier sensitivity: $\left|V_{b l}-V_{b l b}\right|=0.07 \mathrm{~V}$

- driving strength of the precharge circuit scales with (horizontal) array size

The simulation results of the worst case analysis, when using $3 \sigma$ OL error of $8 \mathrm{~nm}$, are shown in Fig. 4. The $x$ axes give the different array sizes and the $y$ axes give the nominal (without patterning variability) $t d$ value along with the variability-induced $t d$ penalty $\left(t d_{p}\right)$ for each option and array size. The worst case of $\mathrm{LE}^{3}$ variability shows up to $\sim 20 \% t d_{p}$. Worst case impact of SADP and EUV is limited and less than $3 \% t d_{p}$ in all cases.

Since this is a worst case analysis, it is important to evaluate 


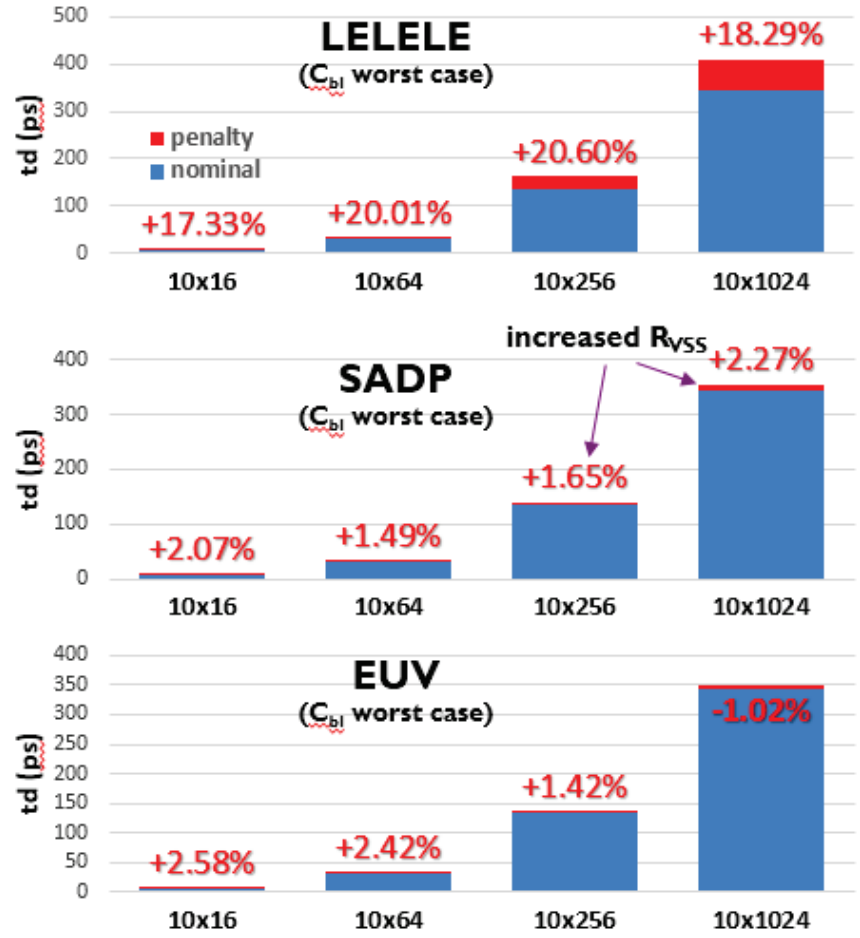

Fig. 4: Worst case wire variability impact on $t d$.

the probability of the worst case occurrence. However, the extraction of statistical distributions of $t d_{p}$ by simulation of full netlists of SRAM arrays, including parasitics, would be extremely time consuming. Furthermore, there is an unclear trend in $t d_{p}$ values for different array sizes: $t d_{p}$ initially increases and later tends to reduce for $\mathrm{LE}^{3}$ and becomes even negative for EUV, as shown in Fig. 4.

These issues are addressed by an analytical formulation of $t d \& t d_{p}$, presented in the next section.

\section{ANALYTICAL FORMULATION \& STATISTICAL DISTRIBUTION OF $t d_{p}$}

The analytical formula to calculate $t d$ should take into account the $R C_{b l}$ variability and the array size as parameters. Then, $t d_{p}$ can be derived as the ratio of: $t d$ including $R C_{b l}$ variability over nominal $t d$ (without $R C_{b l}$ variability), for a given array size.

\section{A. Lumped RC delay formula}

Based on the transient response function of an $\mathrm{RC}$ circuit with a constant input voltage $\mathrm{V}$ [11]:

$$
V_{\text {out }}(t)=\left(1-e^{-t / R C}\right) \cdot V
$$

we can define $t d$ as:

$$
t d=t=a \cdot R C
$$

where $R C$ is the time constant (with $R$ the lumped resistance of the bl net and $C$ the lumped capacitance) and $a$ is a constant defined by the target discharge level. Constant $a$ can be derived from eq. (1) by defining the ratio Vout/V and by solving for $t$. For example, $a$ is 1 for a charging level $V_{\text {out }} \approx 63.2 \%$ of the applied voltage $(V)$. In this case study, for $10 \%$ discharge level, $a$ is calculated as:

$$
0.1=1-e^{-t / R C} \Rightarrow t \approx 0.105 \cdot R C
$$

Breaking $R C$ into the [lumped] resistance and capacitance components and including the length of the array as $n$, the following equation can be derived:

$$
\begin{aligned}
t d=a \cdot\left(n \cdot R_{b l} \cdot R v a r+R_{F E}\right) \\
\cdot\left(n \cdot\left(C_{b l} \cdot C v a r+C_{F E}\right)+C_{p r e}(n)\right)
\end{aligned}
$$

where: $\underline{n}$ is the the bl length as the number of SRAM cells connected in series, $R_{b l}$ is the bl resistance of one SRAM cell, Rvar is the bl resistance variation (induced by the patterning option) expressed in percentage $(1+x \%), R_{F E}$ is the FrontEnd resistance of the 'discharge path' or the resistance of the pull-down NMOS transistors in one cell (constant value), $C_{b l}$ is the total capacitance of the bl net of one SRAM cell, $\overline{\text { Cvar }}$ is the bl capacitance variation (induced by the patterning option) expressed in percentage $(1+x \%), C_{F E}$ is the FrontEnd capacitance or the capacitance at wl pass-gates where the bl is connected to, and $C_{p r e}(n)$ is the capacitance of the precharge circuit which is connected to the bit lines. The value of $C_{\text {pre }}$ is a function of $n$ according to the scaling formula that is used.

If we transform the equation to a general polynomial in $n$ the result is a second-degree-like polynomial of the form:

$$
\begin{aligned}
t d= & n^{2} \cdot\left(a \cdot R_{b l} \cdot \operatorname{Rvar} \cdot\left(C_{b l} \cdot C \operatorname{var}+C_{F E}\right)\right) \\
& +n \cdot\left(a \cdot R_{F E} \cdot\left(C_{b l} \cdot C v a r+C_{F E}\right)\right. \\
& \left.+a \cdot R_{b l} \cdot \operatorname{Rvar} \cdot C_{\text {pre }}(n)\right)+a \cdot R_{F E} \cdot C_{\text {pre }}(n)
\end{aligned}
$$

In this form it can be seen that there is a quadratic dependency on $n$, plus an -almost- linear dependency on $n$, plus an -almostconstant term (loosely depended on $n$ ). Thus, the time penalty $t d_{p}$ is expressed as a rational function of two polynomials: actual $t d$ over nominal $t d\left(t d_{\text {nom }}\right.$ where Rvar, Cvar $\left.=1\right)$. The polynomial nature of $t d_{p}$ and the negative values of $R v a r$ in the worst cases considered, are the primary reasons for the fluctuation in $t d_{p}$ values over various array lengths.

To test the formula we substitute the values of all parameters (obtained from our models) and compare the results with the simulated $t d_{n o m}$ values for the different array lengths $(n)$. Table II shows the results of this test.

TABLE II: Formula versus simulation $t d_{n o m}$ values.

\begin{tabular}{lcc}
\hline Array size & Simulation & Formula \\
\hline $10 \times 16$ & $5.59 E-12$ & $2.09 E-12$ \\
$10 \times 64$ & $30.07 E-12$ & $7.56 E-12$ \\
$10 \times 256$ & $134.62 E-12$ & $30.87 E-12$ \\
$10 \times 1024$ & $344.85 E-12$ & $144.02 E-12$ \\
\hline
\end{tabular}

The results show a deviation between simulation and formula $t d_{n o m}$ values. This deviation is expected since the formula is based on the lumped $R C$ equation, though bl is a distributed line which can be better approximated with the 
Elmore delay. Furthermore, other components are not behaving as lumped elements, like $R_{F E}$, but are considered as lumped in the formula; or are not taken into account at all (though are included in simulation deck), like leakage currents, $R C$ contribution of metal vias, etc.

Yet, if we introduce the same $R C$ variation as in our simulation, the analytical formula gives very similar $t d_{p}$ percentage values with respect to the simulation results. Table III shows the results for the considered worst cases of variability.

TABLE III: Formula versus simulation $t d_{p}$ values (\%) using the worst case variability.

\begin{tabular}{llccc}
\hline Method & Array size & LELELE & SADP & EUV \\
\hline \multirow{4}{*}{ Simulation } & $10 \times 16$ & 17.33 & 2.07 & 2.58 \\
& $10 \times 64$ & 20.01 & 1.49 & 2.42 \\
& $10 \times 256$ & 20.60 & 1.65 & 1.42 \\
Formula & $10 \times 1024$ & 18.29 & 2.27 & -1.02 \\
\hline & $10 \times 64$ & 18.37 & 1.88 & 2.20 \\
& $10 \times 256$ & 20.43 & 1.62 & 2.15 \\
& $10 \times 1024$ & 18.84 & -4.00 & -1.47 \\
\hline
\end{tabular}

The formula gives a good approximation of $t d_{p}$ for $\mathrm{LE}^{3}$ and EUV but not for the SADP case. For $n>64$, the simulation of SADP shows an uptrend of the $t d_{p}$ value (Fig 4), which is not captured by the formula. A possible reason is that for SADP in this design there is an anti-correlation of the $R_{b l}$ and $R_{V S S}$ since CD variation of the first introduces reversed $\mathrm{CD}$ variation to the latter. In the worst case of SADP there is a drop in $R_{b l}$ of $\sim 18 \%$ which introduces an increase in $R_{V S S}$, that is not considered in the formula. Since this $R_{V S S}$ change is negligible for short array lengths, the formula can still approximate $t d_{p}$ correctly for SADP for $n \leq 64$; this is not the case for SADP and longer array lengths.

\section{B. Monte-Carlo distribution of $t d_{p}$}

Using the analytical formula, the statistical distribution of $t d_{p}$ can be easily extracted given the $R C_{b l}$ variation distribution. We use our parameterized tool to extract the $R C_{b l}$ distribution with Monte-Carlo sampling of process variability for each option as input. For the $\mathrm{LE}^{3}$ case we use four values of $3 \sigma$ OL error in the range from $3 n m-8 n m$. An example of the Monte-Carlo distribution of $t d_{p}$ for $8 \mathrm{~nm}$ OL and array size of $10 x 64$ ( $n=64)$, is shown in Fig. 5.

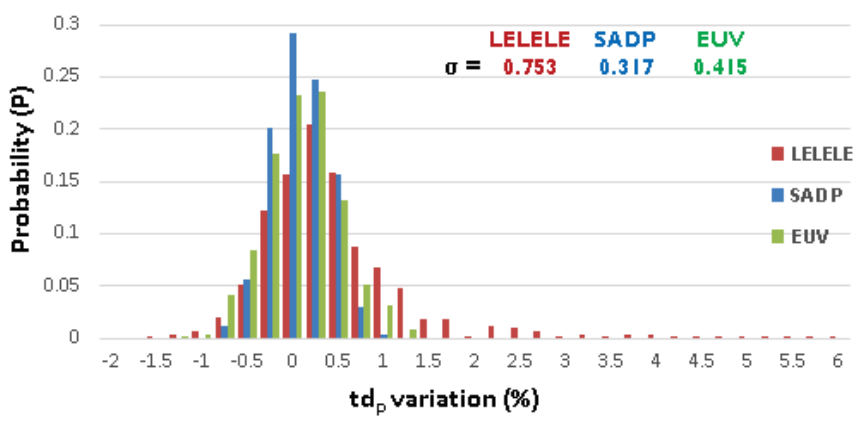

Fig. 5: Monte-Carlo $t d_{p}$ distribution: $8 \mathrm{~nm} 3 \sigma$ OL, $n=64$.

The standard deviation of $t d_{p}$ for $\mathrm{LE}^{3}$ with $8 n m 3 \sigma$ OL is more than double compared with SADP. A summary of all standard deviation values for the three patterning options and for a set of $3 \sigma$ OL values is included in Table IV.

TABLE IV: Patterning options \& $t d_{p} \sigma$ values.

\begin{tabular}{ccc}
\hline Array size & Patterning option & Std. deviation $(\sigma)$ \\
\hline \multirow{6}{*}{$10 \times 64$} & LELELE 3nm OL & 0.414 \\
& LELELE $5 \mathrm{~nm}$ OL & 0.454 \\
& LELELE $7 \mathrm{~nm}$ OL & 0.552 \\
& LELELE 8nm OL & 0.753 \\
SADP & 0.317 \\
EUV & 0.415 \\
\hline
\end{tabular}

The OL error plays a decisive role in $\mathrm{LE}^{3}$ performance impact distribution. Tight OL control, with a $3 \sigma$ error of $3 \mathrm{~nm}$ or less, is required for $\mathrm{LE}^{3}$ to reach similar performance variation values with SADP and EUV. If this tight OL budget cannot be met and if the introduction of EUV in IC manufacturing keeps being postponed, SADP appears to be the optimal MP option, as far as performance variation impact is concerned.

\section{CONCLUSIONS}

In this paper we simulate the performance variation in SRAMs, due to the interconnect variability generated by MP options and EUV. In the worst case scenario of interconnect variability, the read time penalty introduced by the $\mathrm{LE}^{3}$ option can be as high as $\sim 20 \%$, compared to $<3 \%$ for SADP and EUV. The full distribution of the performance variations, obtained from Monte-Carlo simulations based on an analytical model of the read time, confirms the low impact of SADP. The distribution of $\mathrm{LE}^{3}$, on the other hand, shows the higher performance variation, with a standard deviation $(\sigma)$ value as much as double than the other options. The main contributor to this performance variation of $\mathrm{LE}^{3}$ is the exposure overlay (OL) error. Limiting the $3 \sigma$ OL error to $\leq 3 \mathrm{~nm}$ allows $\mathrm{LE}^{3}$ to reach comparable performance variations with respect to SADP and EUV.

\section{REFERENCES}

[1] B. Smith, 'Immersion techniques carry 193nm lithography beyond the $65 \mathrm{~nm}$ node," Rochester Institute of Technology, SPIE's oemagazine, Jul 2004.

[2] C. Fonseca et al., "Advances and challenges in dual-tone development process optimization," Proc. SPIE vol. 7274, 72740I, Mar 2009.

[3] T. Honda et al., "Influence of resist blur on ultimate resolution of ArF immersion lithography," J. Microlith., Microfab., Microsyst., Vol. 5, 043004, Oct-Dec 2006.

[4] "List of leading EUVL technical challenges," 2014 International workshop on EUVL, Makena beach, Maui, Hawaii, Jun 2014.

[5] R. Ghaida, P. Gupta, "Role of design in Multiple Patterning: technology development, design enablement and process Control,' IEEE Design Automation and Test in Europe (DATE), pp. 314 - 319, Mar 2013.

[6] A. Hazleton et al., "Exposure tool requirements for $32 \mathrm{~nm}$ node: Double Patterning and alternatives," International Symposium on immersion lithography extensions, Sept 2008.

[7] D. Abercrombie, "Mastering the magic of Multi-Patterning," Mentor Graphics white paper, Dec 2013.

[8] Nikon eReview, "Lithography experts report on advanced multiple patterning solutions and cost," Spring 2014.

[9] International Technology Roadmap for Semiconductors. [Online]. Available: http://www.itrs.net/Links/2013ITRS/Summary2013.htm.

[10] T. Chiou, M. Dusa, A. Chen, D. Pietromonaco, "Lithographic challenges and their solutions for critical layers in sub-14nm node logic devices," Proc. SPIE vol. 8683, 86830R, Apr 2013.

[11] J.M. Rabaey, "Digital Integrated Circuits: A Design Perspective," second edition, Prentice Hall, 0130909963 978-0130909961, Jan 2003. 